\title{
Kinematics and dynamics of molecular gas in galactic centers
}

\author{
K. Sakamoto \\ Academia Sinica, Institute of Astronomy and Astrophysics \\ Taipei 10617, Taiwan \\ email: ksakamoto@asiaa.sinica.edu.tw
}

\begin{abstract}
The central molecular zone (CMZ) in the central half kpc of the Milky Way is a massive concentration of molecular gas in the center of a barred spiral galaxy. Current and past activities in the Galactic center include the formation of massive stars/clusters, AGN feeding, and feedback. At the same time, observations of molecular gas in external galaxies show that many disk galaxies have similar condensations of molecular gas in their central kpc or so. They also have CMZs, or nuclear molecular rings or concentrations in more common terms among extragalactic observers. The formation of the CMZs are often, but not always, related to stellar bars. The centers of nearby galaxies can provide valuable information on the general properties of galactic centers and CMZs through comparative studies of multiple galactic centers of different characteristics from various viewing angles. Linear resolutions achieved toward nearby extragalactic CMZs with modern radio interferometers are now comparable to those achieved toward the Galactic CMZ with small single-dish telescopes. I review and present work on the formation mechanism and properties of the CMZs in external galaxies with some comparisons with the CMZ of our Galaxy.
\end{abstract}

Keywords. galaxies: ISM — galaxies: nuclei — galaxies: kinematics and dynamics — ISM: molecules

\section{Introduction}

Spiral galaxies often have structures called (circum)nuclear rings in their centers (e.g., Comerón et al. 2010). These rings are seen in young stars, dust lanes, or sometimes molecular line emission - all of these suggest ring-like concentrations of star forming gas and dust. The nuclear rings are found mostly, but not always, in barred spiral galaxies. A causal relation between nuclear rings and orbital families in barred galaxy potentials has long been recognized (e.g., Combes \& Gerin 1985); see Regan \& Teuben (2003) for the nuclear rings being not at the inner Lindblad resonances but still related to the significant presence of so-called $x_{2}$ orbits. Also theoretically predicted is the causal relation between a concentration of gas toward galactic centers and the a stellar bar in the form of bardriven radial gas transport (Sorensen et al. 1976; Athanassoula 1992). At the same time, our Milky Way Galaxy is a barred spiral galaxy and has a strong gas concentration in its center, called the central molecular zone (CMZ) (Blitz \& Spergel 1991; Morris \& Serabyn 1996). The causal relation between the stellar bar of our Galaxy and the CMZ has also long been regarded most likely (Matsuda \& Nelson 1977). The proposed dynamical mechanisms for the formation of nuclear rings in barred galaxies and that for the CMZ of our Galaxy are identical. Therefore it is reasonable to regard the CMZ in our Galaxy and (many) nuclear rings in external galaxies as the same phenomenon.

Putting the Galactic CMZ and extragalactic nuclear rings in a single category, one can start answering questions that could not be answered before. For example, the relation between the Galactic bar and the Galactic CMZ had only a unique case before, but 

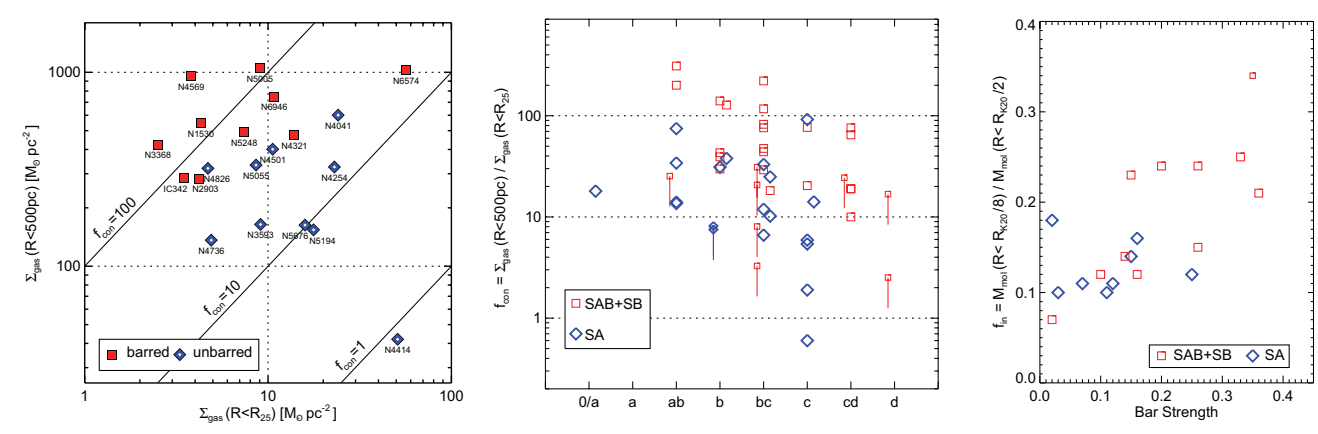

Figure 1. Concentration of molecular gas to galactic centers according to galaxy types. Left: Galaxies are plotted in the parameter space of mean gas surface density in the central kpc (abscissa) and the mean density in the entire optical disk (ordinate). Figure 4 of Sakamoto et al. (1999) is replotted. Middle: A variant of Figure 2 of Sheth et al. (2005) plotting the distribution of gas central concentration indices against galaxy morphologies. The data of Sheth et al. (2005) and Sakamoto et al. (1999b) are shown together. Small symbols with vertical lines are $2 \sigma$ upper limits. Right: Figure 49 of Kuno et al. (2007) showing the relation between degrees of molecular gas central concentration $f_{\text {in }}$ and the bar strengths measured by Laurikainen \& Salo (2002). Two data points in the original figure are either plotted with a small symbol or moved. They are the ones with their bar strengths being $Q_{\mathrm{b}}$ in Table 1 of Laurikainen \& Salo (2002). The moved point now uses $Q_{\mathrm{H}}$ in Table 3 to be consistent with the other points. [A COLOR Version Is AVAilable online.]

is a subject of statistical studies after identifying the Galactic CMZ as a nuclear ring. Also, outside our Galaxy we can observe CMZs from various viewing angles and in galaxies with various properties; neither of which is possible for our Galaxy. At the same time the Galactic CMZ can be observed with the highest linear resolution than possible toward any other nuclear rings. Therefore the Galactic CMZ keeps providing extragalactic observers with examples of features or phenomena to look for whenever a higher spatial resolution than before becomes available. This synergy of Galactic and extragalactic studies of CMZs (or nuclear rings) has become increasingly evident as the linear resolutions achievable for nearby galaxies with radio interferometers have reached the linear resolution available at the Galactic center with single-dish telescopes.

In this contribution, I review studies on the kinematics and dynamics of molecular gas in galactic centers (including the center of our Galaxy) conducted toward the unified understanding of the formation and properties of the galactic and extragalactic CMZs. Also presented are some of my ongoing (and hence preliminary) work made with my collaborators using the Submillimeter Array (SMA) and the Atacama Large Millimeter/submillimeter Array (ALMA). These telescopes, in particular ALMA, is going to be the main workhorse in this area of research.

\section{Bar-driven gas transport: statistics}

Regarding the formation mechanism of CMZs, statistical evidence has been presented for bar-driven transport of molecular gas toward galactic centers by Sakamoto et al. (1999b), Sheth et al. (2005), and Kuno et al. (2007). Their observations are summarized in Figure 1.

Galaxies in Figure 1 (left) are plotted according to their global and central mean gas surface densities. The abscissa is the mean surface density of molecular gas averaged over the optical disk of each galaxy (i.e., $R<R_{25}$ ) and the ordinate is the mean surface density of molecular gas in the central kiloparsec (i.e., $R<0.5 \mathrm{kpc}$ ). The inner averaging area matches the often-seen central gas concentrations and nuclear rings in spiral 
galaxies (Sakamoto et al. 1999a, Camerón et al. 2010). The ratio of the central-to-global surface densities, $f_{\text {con }} \equiv \Sigma_{\text {mol }}(R<0.5 \mathrm{kpc}) / \Sigma_{\text {mol }}\left(R<R_{25}\right)$, is an index of gas central concentration in each galaxy. The concentration index $f_{\text {con }}$ is constant on each diagonal line in the plot, and it is larger (i.e., gas is more concentrated toward the central kpc) in galaxies near the upper-left corner. Galaxies are plotted with different symbols for barred and unbarred morphological types (SAB+SB and SB, respectively, in de Vaucouleurs et al. 1991). It is evident from the data that barred galaxies have higher degrees of gas concentration to their central kpc (Sakamoto et al. 1999b).

Figure 1 (middle) plots the gas concentration index $f_{\text {con }}$ as a function of the Hubble morphological stage (i.e., earlier to the left and later to the right) using the data of Sheth et al. (2005), which is for optically selected galaxies, supplemented with the data of Sakamoto et al. (1999b), which observed CO bright targets. These data confirm that barred galaxies tend to have larger degrees of gas central concentration than unbarred galaxies. Moreover, as Sheth et al. (2005) found and the plot shows, early type spiral galaxies tend to have larger degrees of gas concentration; this has been confirmed by Komugi et al. (2008). Also there exist barred galaxies lacking their central gas concentrations (i.e., barred galaxies with $f_{\text {con }}$ upper limits in the plot.) Sheth et al. (2005) proposed that these barred galaxies had swept all the gas within their bar radii and had consumed the gas at the galactic centers thorough starbursts.

Figure 1 (right) is a slightly revised Figure 49 of Kuno et al. (2007) comparing a gas concentration index against bar strength, which is the maximum tangential force normalized by the mean radial force in the galaxy's gravitational potential. Kuno et al.(2007) found a positive correlation between the bar strength and the degree of gas concentration in these data. In addition they confirmed with their single-dish CO atlas of 40 spiral galaxies the trend that barred galaxies have higher degrees of $\mathrm{CO}$ central concentration than unbarred spirals. The concentration index used here, $f_{\text {in }}$, is different from $f_{\text {con }}$ in that its numerator is not a quantity for a fixed linear radius but for a radius that scales with the galaxy size. Barred spirals have higher values of both concentration indices.

The concentration indices used above are not affected by galaxy-to-galaxy variation, if any, of the ratio of molecular gas mass to the $\mathrm{CO}$ luminosity, or the $X_{\mathrm{CO}}$ factor, because $X_{\mathrm{CO}}$ is in both the denominator and numerator. $X_{\mathrm{CO}}$ is reported to be higher in the centers of many galaxies than in their disks; for example, Bolatto et al. (2013) recommend an $X_{\mathrm{CO}}$ for $R \lesssim 0.5 \mathrm{kpc}$ that is 4 times lower than the $X_{\mathrm{CO}}$ at $1 \mathrm{kpc}$ $\lesssim R \lesssim 9 \mathrm{kpc}$ for our Galaxy. However, even that radial variation does not affect our comparison of the concentration indices between barred and unbarred galaxies as long as the shape of the radial $X_{\mathrm{CO}}$ variation is independent of bars. Regan et al. (2006) used the $8 \mu \mathrm{m}$ emission from polycyclic aromatic hydrocarbons (PAHs) to find that the excess of interstellar medium in the centers of barred galaxies exists also in PAH emission and is hence, in general, not because emissivity of $\mathrm{CO}$ is preferentially enhanced in barred galactic centers.

\section{Bar-driven gas transport and CMZs in M83 and NGC 253}

M83 and NGC 253 provide us with among the best opportunities for detailed case studies of the bar-driven gas transport and the properties of extragalactic CMZs. It is because they are among the nearest barred galaxies with significant central gas concentrations.

\section{1. $M 83$}

Figure 2 shows molecular gas in the stellar bar of M83. There is a pair of molecular gas lanes on the leading sides of the stellar bar, following the dust lanes in the optical image. 

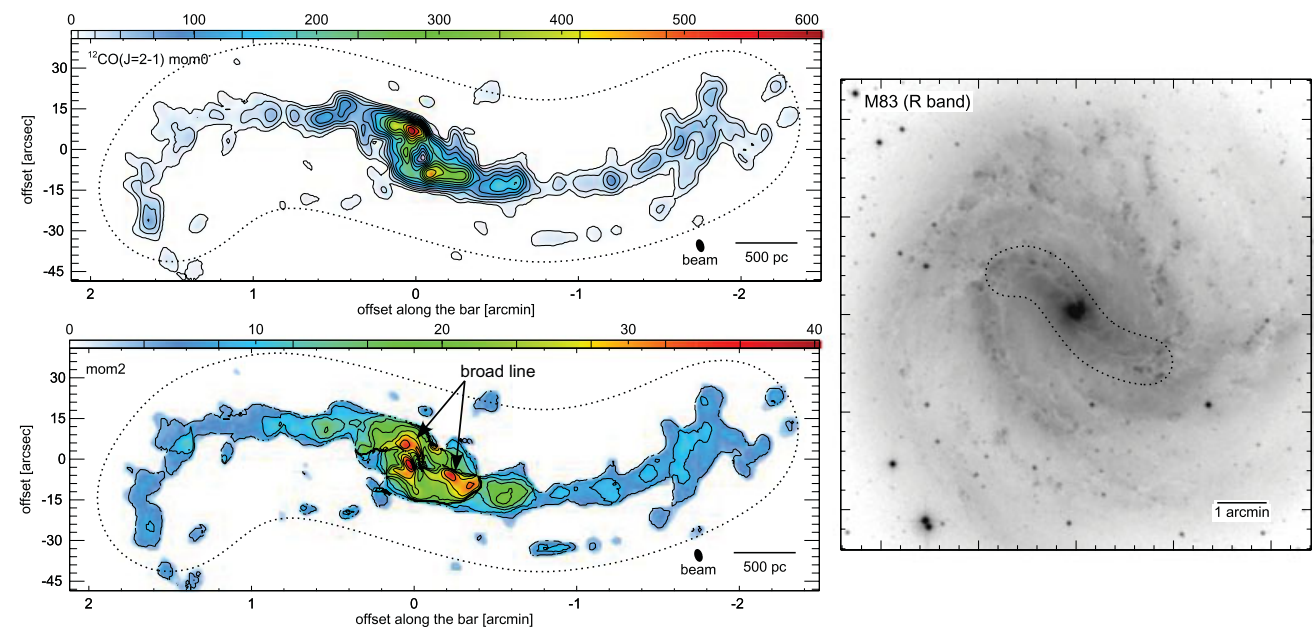

Figure 2. M83 molecular bar. Left top: CO $J=(2-1)$ image showing a pair of gas ridges on the leading sides of the M83 bar as well as the CMZ, which is a nuclear ring with high gas surface densities in the central $\sim 0.5 \mathrm{kpc}$. Left bottom: Distribution of intensity-weighted CO line width, showing regions of large widths in the CMZ. Right: Optical image of M83 from Dale et al. (2009); the CO mapping area is marked. [A COLOR VERSion is aVAILABle online.]

(The galaxy rotates clockwise, assuming trailing spiral arms.) The gas lanes near the galactic center are connected to a circumnuclear ring (Sakamoto et al. 2004). The central $\sim 0.5 \mathrm{kpc}$ of the galaxy including the nuclear ring can be called the central molecular zone of M83 on the basis of its distinctively strong CO emission. The M83 CMZ has two regions of large CO line widths as marked in Figure 2. (The third one at about the origin of the offset coordinates is due to the M83 nucleus and is not discussed here.)

The regions of large CO line widths are at the locations where the gas flows on $x_{1}$ and $x_{2}$ orbits converge or where the gas that was on an $x_{1}$ orbit 'sprays back' to another $x_{1}$ orbit on the other side of the bar after passing the $x_{1}-x_{2}$ convergent point. This is illustrated in Figure 3. The gas orbital motions in this way in a CMZ (or a nuclear ring) has been theoretically predicted (Athanassoula 1992) and suggested from observations of the Galactic CMZ (Binney et al. 1991) and extragalactic nuclear rings (Kenney et al. 1992). The middle panels in Figure 3 show CO line profiles at one of the regions with large line widths. One of the profiles shown clearly has two components and the other can also be decomposed to two components assuming that each component is a Gaussian. The presence of two velocity components is what is expected from the region being a convergent area of two types of gas orbits. Such double-peak line profiles have been observed in the $x_{1}-x_{2}$ orbit-crowding regions of a number of barred galaxies (e.g., Regan et al. 1999; Sakamoto et al. 2007; Hsieh et al. 2011).

It is noteworthy that the large line-widths and double-peak profiles shown in Figure 3 are observed at the size scale of a single giant molecular cloud; $1^{\prime \prime}=22$ pc at the 4.5 Mpc distance of M83. The twin line peaks observed at low resolutions were often attributed to the crowding of the two types of orbits in the beam areas of several $100 \mathrm{pc}$, assuming that at sufficiently high spatial resolution each position has a single velocity as in hydrodynamics of one component fluid (Regan et al. 1999). The new high-resolution data appear to suggest a modification to the previous view so that gas clouds in the ISM gives some particle-ness to the flow. This allows multiple velocity components at the cloud scale and hence collisions between molecular clouds. This is actually an old picture of ISM simulated in e.g., Combes \& Gerin(1985). In this picture, two velocity 

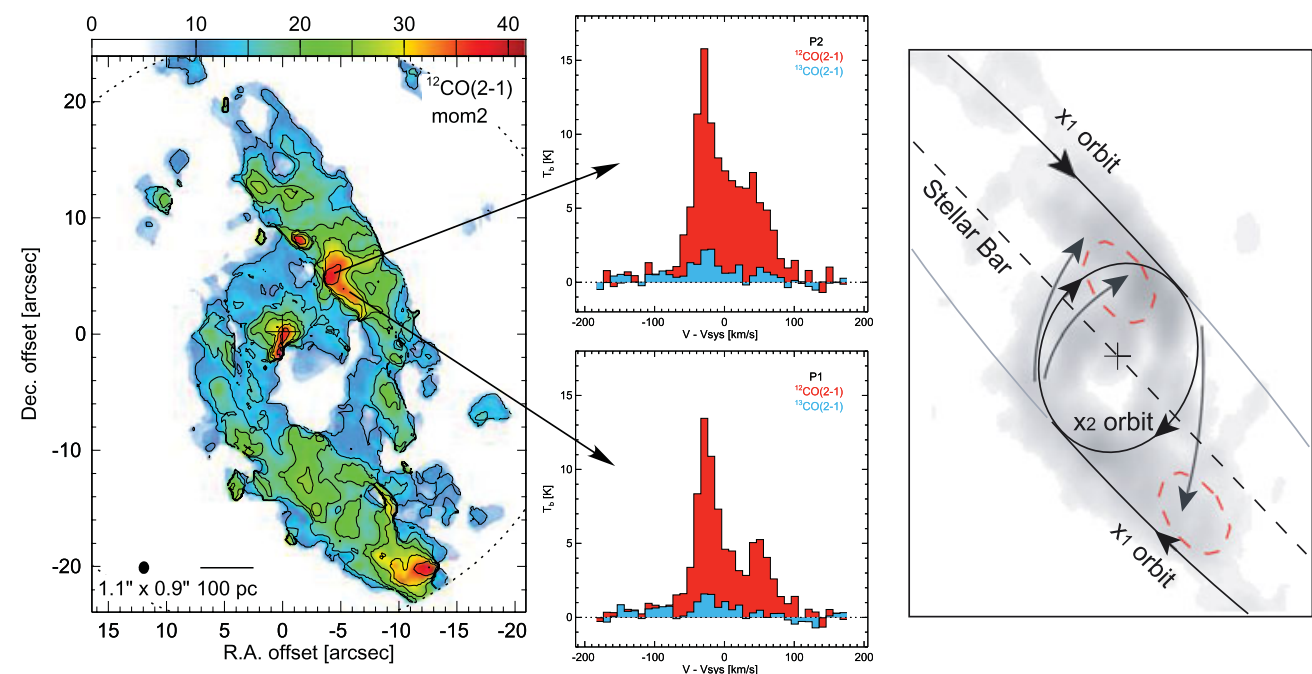

Figure 3. M83 central molecular zone (CMZ). Left: CO $J=(2-1)$ second moment map showing distribution of CO line width. Middle: CO $J=(2-1)$ line profiles sampled from a region of large $\mathrm{CO}$ line widths. The ${ }^{13} \mathrm{CO} J=(2-1)$ line profiles measured at the same locations assure that the two velocity components in ${ }^{12} \mathrm{CO}$ are not artifacts of ${ }^{12} \mathrm{CO}$ self-absorption at the line center. Right: Model gas orbits in the CMZ. [A Color version is available online.]

components can exist downstream a convergent point of two flows for about the length of cloud mean free path. Plausibly as results of cloud collisions and shocks, there are peaks of dense molecular gas and active star formation at or slightly downstream the convergent point (Muraoka et al. 2009; Sakamoto 2013a). Cloud collisions in the CMZ or nuclear rings of galaxies have been indeed postulated for the reason of (sometime) active star formation in those circumnuclear gas concentrations (Kenney et al. 1992; Kohno et al. 1999). For the Galactic CMZ, Hasegawa et al. (1994) proposed a collision of two giant molecular clouds in the Sgr B2 region and suggested it as the trigger of active star formation there.

\section{2. $N G C 253$}

Figure 4 shows in the stellar bar of NGC 253 the distribution of dust (through nearinfrared color excess) and the distribution of molecular gas (through the $1.3 \mathrm{~mm} \mathrm{CO}$ emission line). The dust and gas distributions generally agree well, both showing lanes on the leading sides of the stellar bar and a conspicuous concentration in the central about 500 pc. This is the central molecular zone of NGC 253 (see Sakamoto et al. 2006a and references therein for early interferometric observations of this CMZ.) The overall gas distribution with respect to the bar and the presence of a conspicuous central gas concentration are in line with the models of bar-driven gas dynamics and the trends of bar-driven gas transport to galactic centers.

The CMZ of highly inclined NGC $253\left(i \sim 80^{\circ}\right)$ appears strikingly similar to that of our Galaxy, which is also edge-on. In Figure 5, the top panel shows the CMZ of our Galaxy and the bottom panel the CMZ of NGC 253. Both are in the same CO $J=(2-1)$ line and at the same linear resolution of about $20 \mathrm{pc}$. The Milky Way data are from single-dish observations by Sawada et al. (2001) and NGC 253 data are from interferometric observations (Sakamoto et al. 2011). Comparisons of the two CMZs are, roughly speaking, the following (Sakamoto et al. 2011 and references therein). The center of the starburst galaxy NGC 253 is $\sim 10^{2}$ times more infrared luminous than the Galactic 

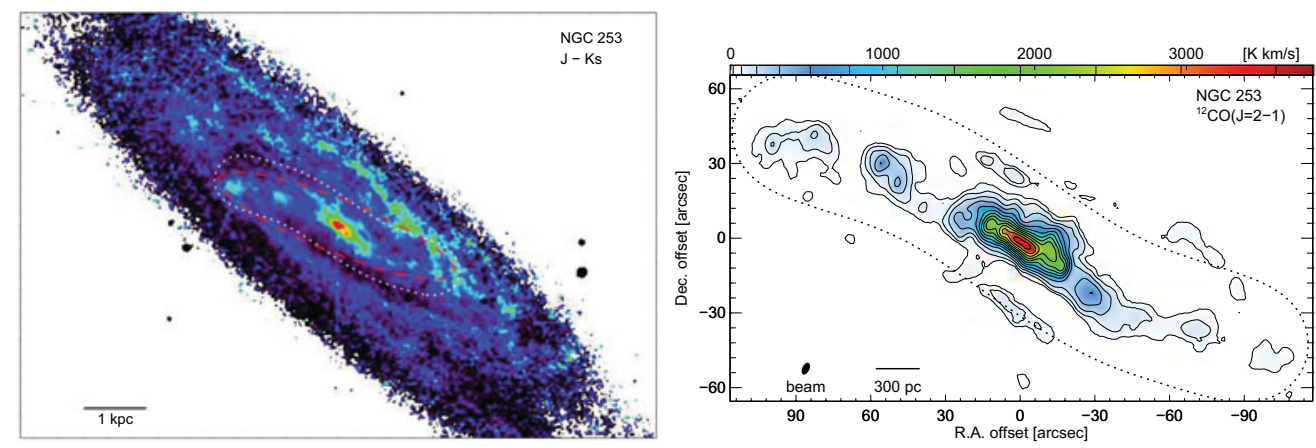

Figure 4. Left: NGC $253 J-K_{\mathrm{s}}$ color index image of NGC 253 made from the 2MASS data of Jarrett et al. (2003). The galactic center has the largest reddening. The dashed ellipse along P. A. $=68^{\circ}$ outlines the stellar bar of this galaxy. The dotted curve shows the area mapped in CO $J=(2-1)$ using the SMA. Right: CO $J=(2-1)$ emission in the bar of NGC 253, showing the prominent central condensation (CMZ) and gas on the dust lanes in the bar. [A COLOR VERSION IS AVAILABLE ONLINE.]

center. They both have several gas clumps of 50-100 pc sizes in these maps; one of them in the Galactic center is the Sgr B molecular cloud complex. The peak CO integrated intensities at those clumps are about a factor of three larger in the CMZ of NGC 253. This may be because the CMZ gas clumps in the starburst nucleus has larger gas column densities, but a firm conclusion needs a better knowledge about the CO-to- $\mathrm{H}_{2}$ conversion factors in these CMZs (in particular, whether they are the same in the two CMZs of different star formation intensities.) An indication of different molecular gas properties between the two CMZs is that the peak beam-averaged CO brightness temperature is a factor of 3 larger in NGC $253(50 \mathrm{~K})$ than in the Milky Way CMZ $(17 \mathrm{~K})$.

Another notable difference between the CMZs of the Milky Way and NGC 253 is that the latter with much more active star formation shows more signs of feedback from the forming stars to the interstellar medium. A 100 pc diameter cavity is evident in Figure 5 on the northeastern edge of the CMZ. More cavities and possible ones have been reported in this CMZ although they need careful confirmation because a possible cavity found in a low-resolution image can turn out to be more complex, non-circular structure in higher resolution followup (Sakamoto et al. 2006, 2011; Bolatto et al. 2013). Moreover, Bolatto et al. (2013) recently imaged molecular gas that is outflowing from the CMZ perpendicular to the galactic plane. A spur to the southwest of the nucleus in Figure 5 is a part of that. Earlier reports of a molecular outflow from the center of NGC 253 are in Turner (1985) and García-Burillo et al. (2000). Although the CMZ of our Galaxy has smaller molecular cavities (Oka et al. 2012) and also has a sign of a large scale outflow or explosive events (Murakami et al. 2000; Su et al. 2010), molecular gas structures of the magnitudes of these features are not in our Galactic CMZ. This is therefore another example of the virtue of studying extragalactic CMZs.

\subsection{Other galaxies}

There are a number of other galaxies, whether barred or unbarred, that have a central concentration of molecular gas that can be arguably called the central molecular zone. A notable class among them is the merging or strongly interacting galaxies in which the dynamical disturbance leads to bar formation and rapid gas inflow toward the merging galactic centers (Barnes \& Hernquist 1991, 1996). Those merger-induced CMZs often have vigorous star formation and feedback to the ISM in the form of molecular outflow, as seen in a smaller scale in the bar-driven CMZ of NGC 253. Figure 6 shows an example 


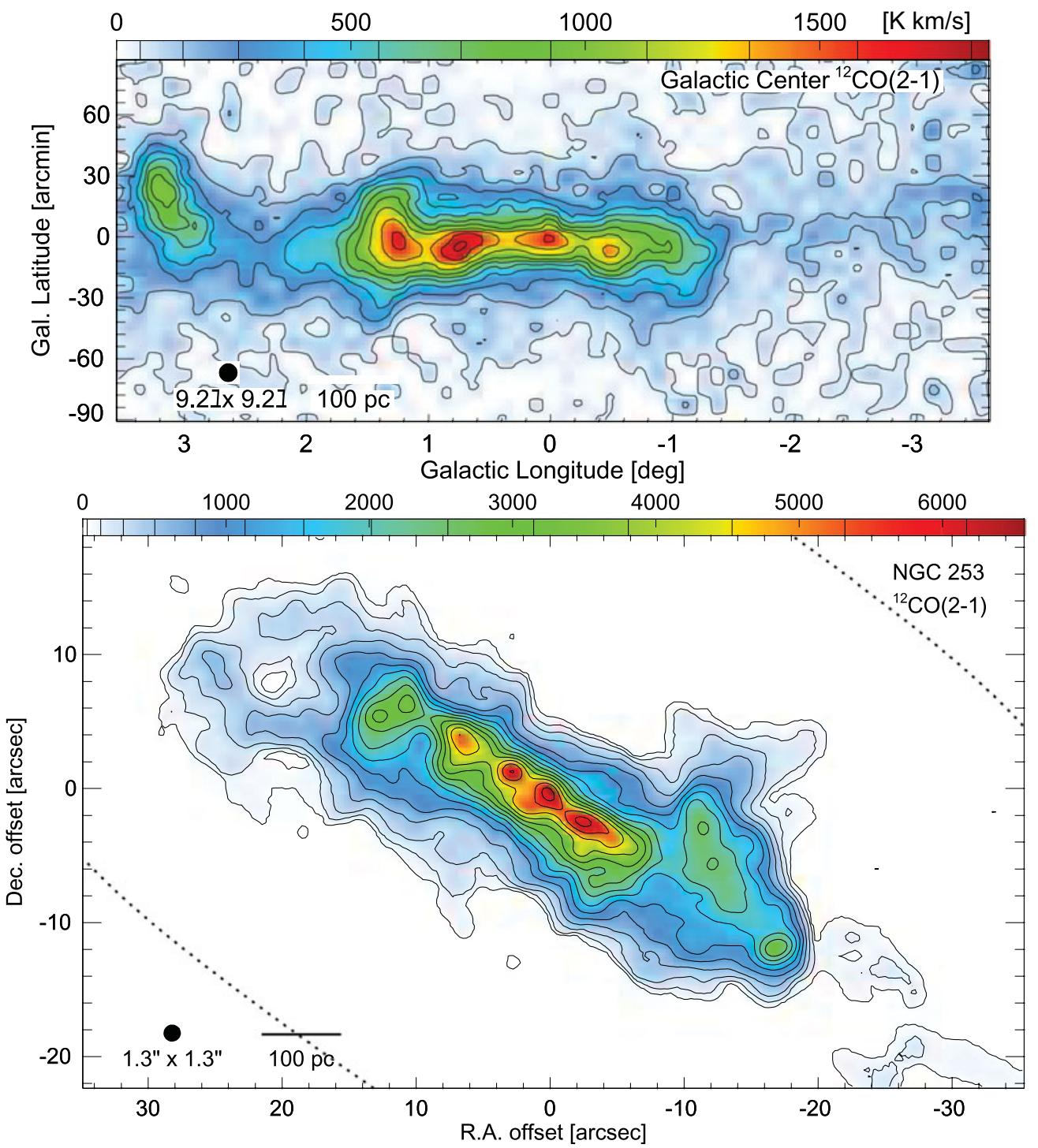

Figure 5. The central molecular zones of the our Galaxy (top) and NGC 253 (bottom). The two are compared here in the same linear scale and linear resolution with the same molecular line, $\mathrm{CO} J=(2-1)$. Integrated intensities are in units of $\mathrm{K} \mathrm{km} \mathrm{s}^{-1}$ in both images. The Galactic center wide-area map was obtained with the University of Tokyo $60 \mathrm{~cm}$ radio telescope (Sawada et al. 2001). NGC 253 was observed with the SMA using up to $300 \mathrm{~m}$ baselines, resulting in a matching linear resolution for a target about 400 times more distant (Sakamoto et al. 2011). [A COLOR VERSION IS AVAILABLE ONLINE.]

taken from recent ALMA observations of a merging luminous infrared galaxy NGC 3256 (Sakamoto et al. in prep.). A concentration of molecular gas (i.e., CO emission) with a FWHM size on the order of a half kpc is seen at each of the two merger nuclei. The peak CO integrated intensities there are no less than that in the CMZ of NGC 253 considering the different linear resolutions. This merger has high-velocity CO emission suggesting a molecular outflow (Sakamoto et al. 2006b, 2013b). Although we can not yet image these distant CMZs at the linear resolutions of a few 10 pc as we could for M83 and NGC 


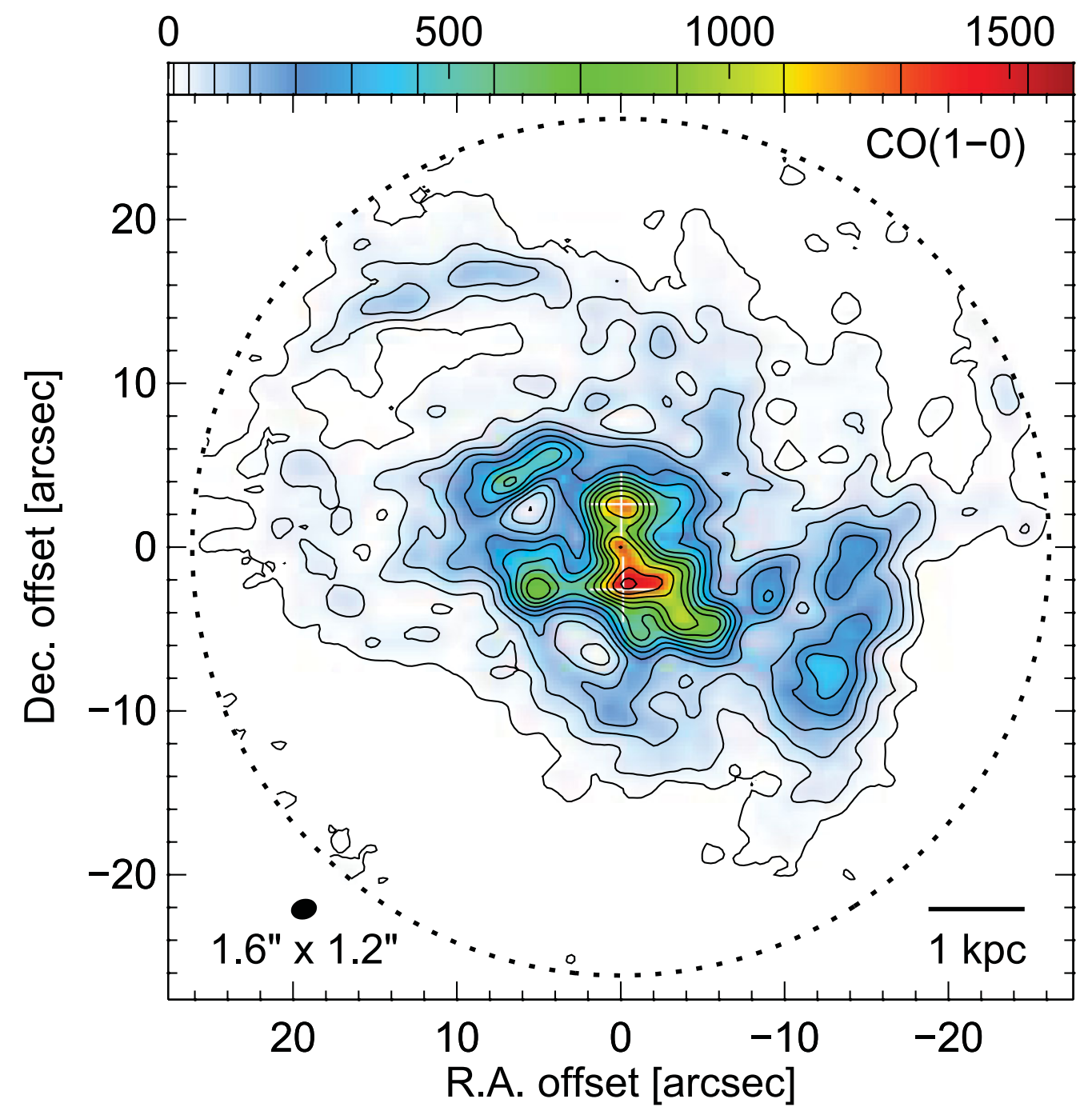

Figure 6. Molecular gas in the merging luminous infrared galaxy NGC 3256 observed with ALMA. The two white plus sings are at the two merger nuclei. The map unit is $\mathrm{K} \mathrm{kms}^{-1}$. Molecular gas concentrations there can be regarded as merger-driven CMZs. [A COLOR VERsion is AVAILAbLE ONLINE.]

253, that is within the reach of ALMA after its completion. Our comparative studies of molecular gas kinematics, dynamics, and properties in galaxy centers can be extended to even these sources in near future.

\section{References}

Athanassoula, E. 1992, MNRAS 259, 345

Barnes, J. E., \& Hernquist, L. E. 1991, ApJ 370, L65

Barnes, J. E., \& Hernquist, L. 1996, ApJ 471, 115

Binney, J., Gerhard, O. E., Stark, A. A., Bally, J., \& Uchida, K. I. 1991, MNRAS 252, 210

Blitz, L. \& Spergel, D. N. 1991, ApJ 379, 631

Bolatto, A. D., Wolfire, M., \& Leroy, A. K. 2013, ARA\&A 51, 207 
Bolatto, A. D., Warren, S. R., Leroy, A. K., et al. 2013, Nature 499, 450

Combes, F., \& Gerin, M. 1985, A\&̈A 150, 327

Comerón, S., Knapen, J. H., Beckman, J. E., et al. 2010, MNRAS 402, 2462

Dale, D. A., Cohen, S. A., Johnson, L. C., et al. 2009, ApJ 703, 517

de Vaucouleurs, G., de Vaucouleurs, A., Corwin, H. G., Jr., et al. 1991, Third Reference Catalogue of Bright Galaxies

García-Burillo, S., Martín-Pintado, J., Fuente, A., \& Neri, R. 2000, A\&\&A 355, 499

Hasegawa, T., Sato, F., Whiteoak, J. B., \& Miyawaki, R. 1994, ApJ , 429, L77

Hsieh, P.-Y., Matsushita, S., Liu, G., et al. 2011, ApJ 736, 129

Jarrett, T. H., Chester, T., Cutri, R., Schneider, S. E., \& Huchra, J. P. 2003, AJ 125, 525

Kenney, J. D. P., Wilson, C. D., Scoville, N. Z., Devereux, N. A., \& Young, J. S. 1992, ApJ 395, L79

Kohno, K., Kawabe, R., \& Vila-Vilaró, B. 1999, ApJ 511, 157

Komugi, S., Sofue, Y., Kohno, K., et al. 2008, ApJS 178, 225

Kuno, N., Sato, N., Nakanishi, H., et al. 2007, PASJ 59, 117

Laurikainen, E., \& Salo, H. 2002, MNRAS 337, 1118

Matsuda, T. \& Nelson, A. H. 1977, Nature 266, 607

Morris, M. \& Serabyn, E. 1996, ARA\&A 34, 645

Murakami, H., Koyama, K., Sakano, M., Tsujimoto, M., \& Maeda, Y. 2000, ApJ 534, 283

Muraoka, K., Kohno, K., Tosaki, T., et al. 2009, PASJ 61, 163

Oka, T., Onodera, Y., Nagai, M., et al. 2012, ApJS 201, 14

Regan, M. W., Sheth, K., \& Vogel, S. N. 1999, ApJ 526, 97

Regan, M. W., \& Teuben, P. 2003, ApJ 582, 723

Regan, M. W., Thornley, M. D., Vogel, S. N., et al. 2006, ApJ 652, 1112

Sakamoto, K., Okumura, S. K., Ishizuki, S., \& Scoville, N. Z. 1999a, ApJS 124, 403

Sakamoto, K., Okumura, S. K., Ishizuki, S., \& Scoville, N. Z. 1999b, ApJ 525, 691

Sakamoto, K., Matsushita, S., Peck, A. B., Wiedner, M. C., \& Iono, D. 2004, ApJ 616, L59

Sakamoto, K., Ho, P. T. P., Iono, D., et al. 2006a, ApJ 636, 685

Sakamoto, K., Ho, P. T. P., \& Peck, A. B. 2006b, ApJ 644, 862

Sakamoto, K., Ho, P. T. P., Mao, R.-Q., Matsushita, S., \& Peck, A. B. 2007, ApJ 654, 782

Sakamoto, K. 2013a, IAU Symposium, 292, 143

Sakamoto, K. 2013b, Astronomical Society of the Pacific Conference Series, 477, 21

Sawada, T., Hasegawa, T., Handa, T., et al. 2001, ApJS 136, 189

Sheth, K., Vogel, S. N., Regan, M. W., Thornley, M. D., \& Teuben, P. J. 2005, ApJ 632, 217

Sorensen, S.-A., Matsuda, T., \& Fujimoto, M. 1976, Ap\&SS 43, 491

Su, M., Slatyer, T. R., \& Finkbeiner, D. P. 2010, ApJ 724, 1044

Turner, B. E. 1985, ApJ 299, 312 